\title{
Descriptive Study of an Outbreak of Avian Urolithiasis in a Large Commercial Egg Complex in Algeria
}

\author{
Hicham SID, Amine FETTAH, Abdelaziz LOUNAS
}

University of Blida, Department of Veterinary Sciences, Blida Road Of Soumaa BP 270 Blida, Algeria; sidhicham@gmail.com

\begin{abstract}
Avian urolithiasis is one of the major causes of mortality in poultry. However, in Algeria this condition has never been described. An outbreak of avian urolithiasis was observed on a large commercial egg complex in the department of Chlef(West of Algeria). The clinical features of this condition are to be described. Mortality associated to urolithiasis started at the onset of egg production, estimated to $0.7 \%$ per week. Urolithiasis induced an egg drop estimated to $12 \%$. Dead and live layers were both necropsied and examined for kidney lesions. Most of the birds examined presented enlarged ureters, renal atrophy and visceral gout deposition.
\end{abstract}

Keywords: Algeria, caged layers, mortality, outbreak, urolithiasis

\section{Introduction}

The development of poultry industry in Algeria has faced serious obstacles of various types, especially on the basis of intensive production systems. Infections of flocks are becoming more often with significant losses, such as avian urinary tract disease. This condition is generally just one element of a systemic bacterial, fungal, viral, or metabolic disease (Styles and Phalen, 1998).

The kidneys are involved in a wide range of essential functions; therefore it is not surprising that kidney dysfunction can cause profound problems for the poultry industry. These problems range in severity from increased manure moisture to catastrophic flock mortality.

Urolithiasis is the formation of large urate "stones" in the ureters of affected birds (Wideman et al., 1983). Death occurs due to uricemia when functional kidney mass is insufficient to sustain a normal metabolism (Mallinson et al., 1984; Randall et al., 1977; Raymond et al., 1988). This condition is primarily observed in caged layers and induces an increased mortality and a decrease in egg production; it has been described in Great Britain as an enzootic disease (Siller, 1981).

Kidney malfunction is associated to multiple etiologies (Darbyshire, 1979; Fitz-Coy et al., 1988; Wideman et al., 1983). Ansar et al. (2004) reported that the urinary system of the treated birds with high dietary calcium and low phosphorous displayed inflammatory lesions, showing abnormalities of color, size, shape and texture of kidneys and ureters. Julian (1982) noticed that water deprivation is a cause of renal disease in chickens. On the other hand, Chousalkar et al. (2007), Ratanasethakul and Cumming, (1983), Mallinson et al. (1984) reported that the nephropathogenic IBV (Infectious Bronchitis Virus) plays an im- portant role in kidney damage. However, in Algeria, this condition has never been reported in the past. This work is a descriptive study that reports an outbreak of urolithiasis in a flock of ISA Brown caged layers in Algeria.

\section{Materials and methods}

\section{Case history}

This study was conducted in a laying hens complex located in Chlef (West of Algeria), housing 270.000 caged layers. Layers were reared in 6 modern industrial houses. Every data was registered since the creation of this complex in 2001 (weekly mortality, treatments, egg production and feed consumption).

Between 2008 and 2010, urolithiasis started to spread from primary flock to all other flocks. It has been noticed a higher mortality rate at the onset of egg production.

This work has been carried out from May 2010 to August 2010. A research group from Blida University (Algeria) investigated this outbreak of avian urolithiasis in a flock of forty five thousand hens of ISA Brown (19 to 35 weeks-old), in order to describe the clinical features and to discuss the possible cause(s) of this condition.

All chicks in the studied flock were from the same source. They were vaccinated in the hatchery for Marek's disease by injection. At day 1 , they were vaccinated in the farm against Infectious Bronchitis (IB) and Newcastle Disease (ND) via spray (Nobilis ND Clone 30+MA5). At day 18, against Gumboro disease via the drinking water (Nobilis Gumboro E228). At 4 and 8 weeks of age via spray, boosters ND and IB were given (Nobilis ND Clone $30 \mathrm{ND}+\mathrm{MA} 5)$. At 10 weeks of age, pullets were vaccinated against Fowlpox via wingweb transfixion. 
At 16 weeks of age, they were vaccinated against EDS (Egg Drop Syndrome), ND and IB by injection of an attenuated vaccine (Nobilis ND+IB+EDS).

The flock was treated during the onset of egg production by two antibiotics (Enrofloxacin then Tylosin) for suspected RCD (Respiratory Chronic Disease). All treatments were added in the drinking water.

\section{Management conditions}

Single age birds were housed in a closed house with application of an all-in-all-out system. The house is built on well-drained land and has natural air movement. In order to ensure that birds receive the ideal amount of fresh air; a tunnel ventilation system is used. Ventilation, cooling and heat systems are controlled by a climate computer. Hot air generators are installed inside the building and uniformly distributed along its length.

Feed is stocked in silos outside the building. The silo can be loaded from above. Inside the house, feed is distributed by automatic hoppers which are controlled by a computer.

Before the transfer of pullets to the production site, all vaccinations were given a week before this operation. Transfer has been carried out at 17 weeks of age; each pullet was housed in less than $350 \mathrm{~cm}^{2}$ floor space and able to reach at least 2 automatic drinkers ( 5 to 6 hens were housed per cage).

Forty five thousand hens were housed in this modern house; all environmental factors were controlled (lighting, temperature, humidity and level of toxic gases in the air). During the outbreak of urolithiasis, performance data were recorded (egg production, feed consumption and body weight). Pullet and layer feed formulations were examined carefully for their nutrient levels and analyzed for mycotoxins levels. The feed is locally prepared for all flocks with changes in nutrient levels for each phase of development. Vaccination program and medications were recorded.

Samples of water and feed from pullet and layer houses were collected for their concentration of heavy metals and calcium-phosphorous levels.

\section{Necropsy observations}

Dead layers (562 layers) aged between 19 to 35 weeksold were necropsied on many occasions during the outbreak of urolithiasis.

Thirty three live layers were randomly selected (one layer per cage and one cage per 200 cages); they were euthanized by dislocation of cervical vertebrae and necropsied. Both live and dead layers were evaluated for the ureters and kidney lesions in the same period (19 to 35 weeks). Depending on the severity of lesions, kidney atrophy was evaluated from slight to severe.

\section{Statistical analysis}

Test of association was made by means of a chi-square test depending on the number of dead or live hens examined; it was conducted at $\mathrm{P}<0.05$.

\section{Results and discussion}

Despite some differences in the age of the affected birds, necropsy observations and case history of this urolithiasis outbreak were similar to urolithiasis in caged layers described in United States (Brown et al., 1987; Cowen et al., 1986, Mallinson et al., 1984). Authors investigated the pathology of same urolithiasis outbreak in caged layers. A variety of agents have been implicated, especially infectious bronchitis viruses and excessive dietary calcium

At the outbreak of urolithiasis, clinical signs at the affected birds were dehydration, pale combs and depression. It is probable that some hens died rapidly without prodromic signs. Some birds exhibited other signs like emaciation, swollen feet; additionally, they had a tendency to hide. These findings are in support with the observations of Fitz-Coy et al. (1988) who reported the same clinical observations in commercial white leghorn pullets.

The mortality at flock of 19 to 35 weeks old due to urolithiasis was relatively high, estimated to $11 \%$ compared to the normal rate (around $1.5 \%$ ). Weekly mortality was estimated to $0.7 \%$ which is in agreement to Brown et al. (1987) who stated that losses from this disease range between 0.5 to $1 \%$ per week. Mortality was mostly associated to severe kidney atrophy and obstruction of ureters.

Necropsy findings for dead and live layers showed kidneys atrophy (Tab. 1) and uroliths deposition. The relationship between renal atrophy and uroliths deposition is showed in Tab. 2. Most of the examined birds presented enlarged ureters due to uroliths (Fig. 1). Several hens presented enlarged pale kidneys (Fig. 2), visceral gout and articular gout in the tibiotarsal joints.

Necropsy observations revealed that kidney atrophy in live chickens was slighter (24\%) than severe (9\%). On the other hand, $45 \%$ of the dead chickens presented severe kidney atrophy. Significant association have been found $(\mathrm{P}<0.05)$ between severe kidney atrophy in dead chickens $(146 / 253)$ and the presence of uroliths which suggests the possibility of the implication of kidney atrophy in uroliths formation. However, most of the live chickens necropsied

Tab. 1. Kidney lesions of caged layers during the urolithiasis outbreak

\begin{tabular}{cccccc}
\hline & \multicolumn{5}{c}{ Kidney atrophy } \\
\cline { 2 - 5 } & Severe & Moderate & Slight & Normal kidney & Number of chickens examined \\
\cline { 2 - 5 } Dead chickens & $253(45 \%)$ & $151(27 \%)$ & $102(18 \%)$ & $56(10 \%)$ & $562(100 \%)$ \\
Live chickens & $3(9 \%)$ & $5(16 \%)$ & $8(24 \%)$ & $17(51 \%)$ & $33(100 \%)$ \\
\hline
\end{tabular}


24

Tab. 2. Severity of kidney atrophy and its relationship with uroliths formation

\begin{tabular}{|c|c|c|c|}
\hline & \multicolumn{3}{|c|}{ Kidney atrophy } \\
\hline & Severe & Moderate & Slight \\
\hline \multicolumn{4}{|c|}{ Dead chickens } \\
\hline Uroliths & $146 / 253$ & $128 / 151$ & $0 / 102$ \\
\hline No Uroliths & $107 / 253$ & $23 / 151$ & $0 / 102$ \\
\hline \multicolumn{4}{|c|}{ Live chickens } \\
\hline Uroliths & $3 / 3$ & $3 / 5$ & $3 / 8$ \\
\hline No Uroliths & $0 / 3$ & $2 / 5$ & $5 / 8$ \\
\hline
\end{tabular}

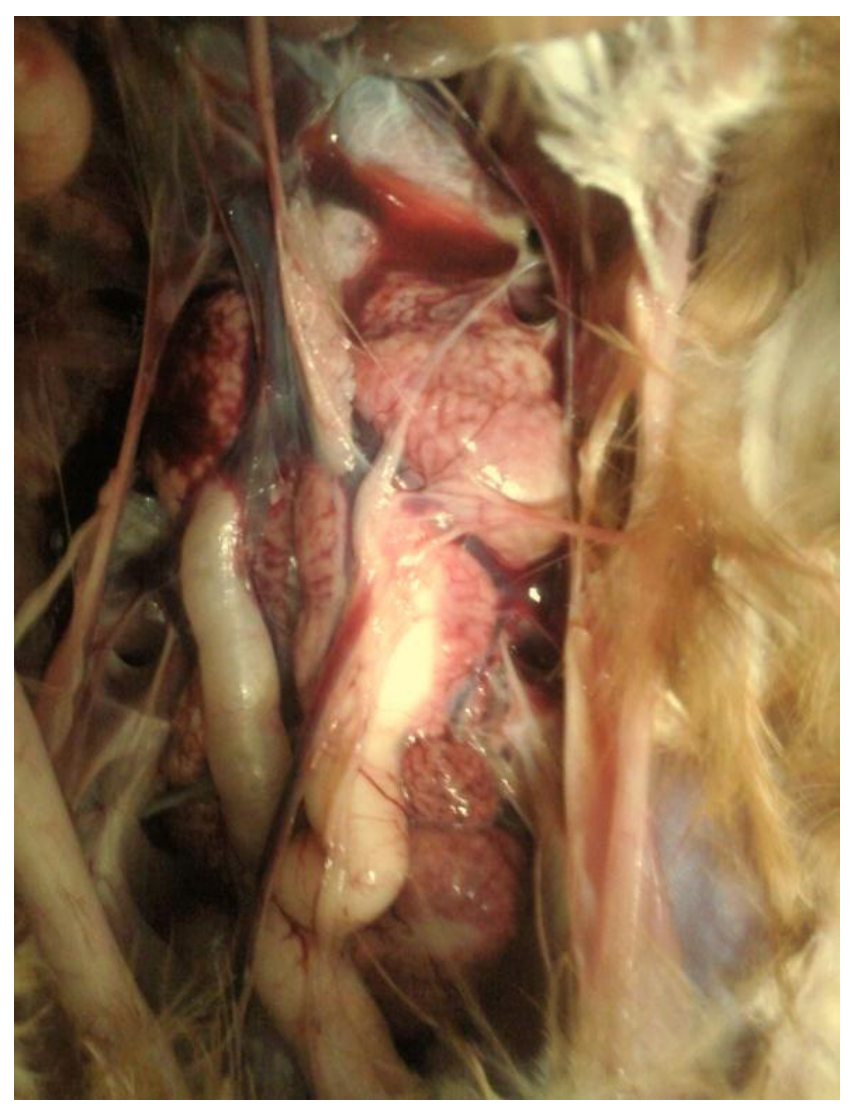

Fig. 1. Live 30 weeks-old layer. Large uroliths filling both ureters with moderate atrophy of the right kidney

presented a slight kidney atrophy (24\%). Our results coincided with the findings of (Siller, 1981) who reported that kidney atrophy contribute to the formation of uroliths.

Feed analysis revealed normal calcium and phosphorus levels. However, it has been noticed that pre-layer and layer formulas contain higher levels of calcium (respectively $2 \%$ and $3.5 \%$ ) than the growing formula (1\%), as this high level is needed for egg production. We have noticed that mortality triggered just after replacing the growing formula by the pre-layer formula. Our results coincided with the findings of Wideman and Cowen (1987); Ansar et al. (2004) who reported that high calcium diets may play an important role in urolithiasis outbreak and modifications of feed formulas can be a factor of physiological stress.
Cage density was relatively high (less than $350 \mathrm{~cm}^{2}$ per layer) which may play the role of stress factor.

Water samples showed acceptable limits of heavy metals concentrations. However, it has been noticed an increase in water intake during this outbreak. According to Julian (1982), water deprivation possibly contributed to outbreaks of urolithiasis in two flocks of laying hens by decreasing fluid flow through the kidneys. Additionally, electrolyte balance may be etiologically involved, as is suggested by the apparent role of water deprivation in outbreaks of urolithiasis and in increased serum urea and uric acid levels (Losing, 1980).

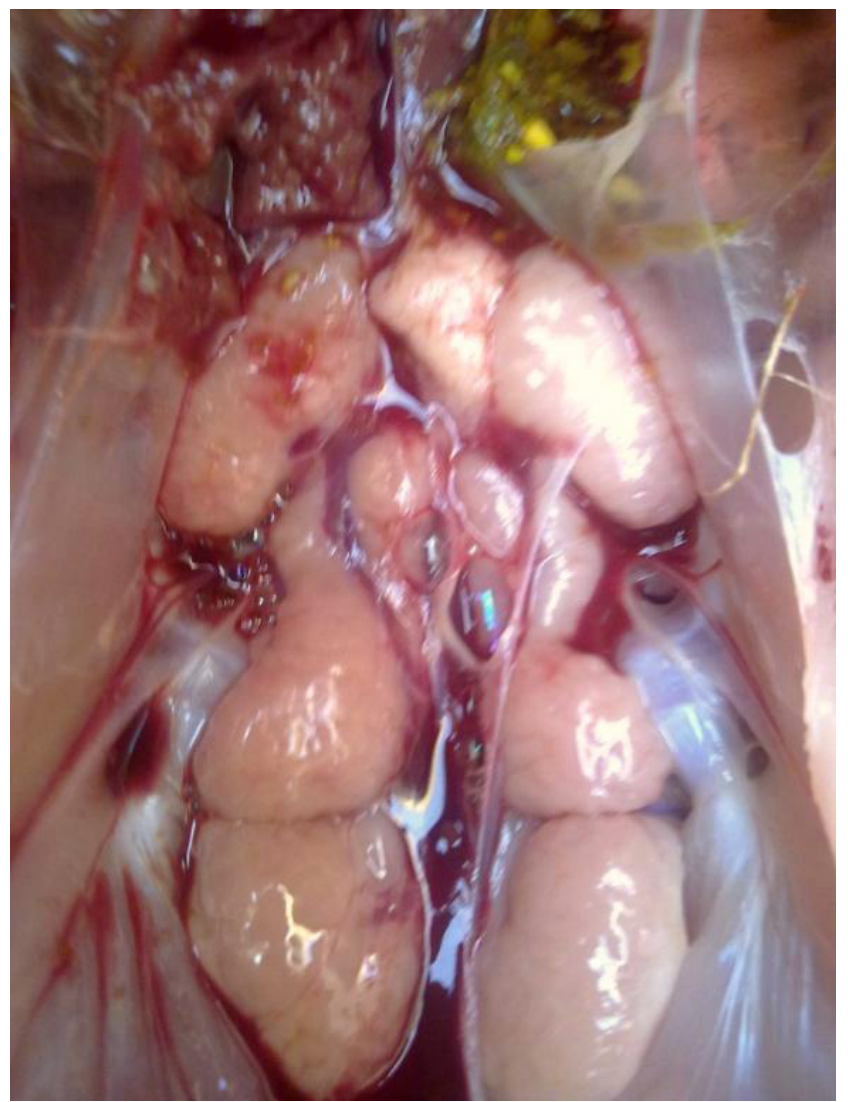

Fig. 2. Live 20 weeks-old layer. Enlarged pale kidneys

Egg production was affected during this outbreak; the egg drop was estimated to $12 \%$. This result doesn't correlate to the observations of (Cowen et al., 1986) who reported that layers affected by urolithiasis remain productive.

During the outbreak, urolithiasis was observed in caged layers 19 or more weeks of age, but later this condition was seen in pullets too (14 to 18 weeks of age).

Despite vaccination program for IBV (Infectious Bronchitis Virus), a variant nephrotropic IBV may be involved in this urolithiasis outbreak. Results of similar outbreaks in Unites States (Cowen et al., 1986; Brown et al., 1987) showed that variant nephrotropic IBV may be involved in kidneys damage. Lee (2002) found that nephropathogenic strains of IBV cause lesions in the kidney, based on several tissue samples collected at various times. 


\section{Conclusions}

Urolithiasis is a degenerative renal disease involving urolith formation in the ureters of caged layers. It may be associated with multiple etiologies (high dietary calcium, low dietary phosphorous or nephropathogenic IBV). In Algeria this condition has never been described before the current study. As a conclusion, we can say that urolithiasis occurs in caged layers at the onset of egg production. Mortality caused by urolithiasis outbreak during this study was important ( $0.7 \%$ per week). The egg drop induced by this condition was estimated to $12 \%$.

\section{References}

Ansar M, Khan SA, Chaudhary ZI, Mian NA, Tipu MY, Rai MF (2004). Effects of high dietary calcium and low phosphorus on urinary system of broiler chicks. Pakistan Vet J 24(3):113-116.

Brown TP, Glisson JR, Gregorio R, Villegas P, Davis RB (1987). Studies of avian urolithiasis associated with an infectious bronchitis virus. Avian diseases 31:629-636.

Chousalkar K, Roberts R, Reece R (2007). Comparative histopathology of two serotypes of infectious bronchitis virus ( $\mathrm{T}$ and N1/88) in laying hens and cockerels. Poultry Science 86:50-58.

Cowen BS, Wideman RF, Rothenbacher BH, Braune MO (1986). An Outbreak of Avian Urolithiasis on a Large Commercial Egg Farm.

Darbyshire JH (1979). Assessment of cross-immunity in chickens to strains of avian infectious bronchitis virus using tracheal organ cultures. Avian Pathol 9:179-183.

Fitz-Coy SH, Edgar A, Hoerr FJ (1988). An Outbreak of Urolithiasis in Single-Comb White Leghorn Pullets. Avian diseases 32:563-566.
Julian R (1982). Water deprivation as a cause of renal disease in chickens. Avian Pathol 11:615-617.

Lee CW (2002). Pathogenisis of infectious bronchitis virus with different routes of inoculation and the effect of in vivo serial passage in nephropathogenicity using cloacal infection. 137th AVMA Annual Convention. Salt Lake City, Utah.

Losing W (1980). Clinical symptoms and diagnosis of drinking water deficiency in chickens. Inaugural Dissertation, Tierarztliche Hochschule, Hannover, West Germany.

Mallinson E, Rothenbacher TH, Wideman RF, Snyder DB, Zuckennan AJRE, Davidson JP (1984). Epizootiology, pathology, and microbiology of an outbreak of urolithiasis in chickens. Avian Dis 28:25-43.

Randall C, Blandford E, Borland N, BrooksbankV, Hall C, Richards $S$ (1977). A survey of mortality in 51 caged laying flocks. Avian Pathol 6:149-170.

Ratanasethakul C, Cumming R (1983). The effect of route of infection and strain of virus on the pathology of Australian infectious bronchitis virus. Aust Vet J 60:209-213.

Raymond P, Glahn RF, Wideman R, Cowen BS (1988). Effect of Dietary Acidification and Alkalinization on Urolith Fonnation and Renal Function in Single Comb White Leghorn Laying Hens. Poultry Science 67:1694-1701

Siller W (1981). Renal pathology of the fowl-a review. Avian Pathol 10:187-262.

Styles D, Phalen D (1998). Seminars in Avian and Exotic Pet Medicine. Clinical Avian Urology 7(2):104-113.

Wideman RF, Mallinson T, Rothenbacher HA (1983). Kidney function in pullets and laying hens during outbreaks of urolithiasis. Poultry Sci 62:1954-1970.

Wideman RF, Cowen BS (1987). Effect of dietary acidification on kidney damage caused by excess calcium and infectious bronchitis virus. Poultry Sci 66:626-633. 